\title{
Pulsation in pre-main sequence stars
}

\author{
Konstanze Zwintz \\ Institute for Astro- and Particle Physics, University of Innsbruck \\ Technikerstrasse 25/8, A-6020 Innsbruck, Austria \\ email: konstanze.zwintz@uibk.ac.at
}

\begin{abstract}
Asteroseismology has been proven to be a successful tool to unravel details of the internal structure for different types of stars in various stages of evolution well after birth. We can now show that it has similar power for pre-main sequence (pre-MS) objects.

Pre-MS stars with masses between $\sim 1$ and 6 solar masses that have recently been formed and gain their energy mainly from gravitational contraction can become vibrationally unstable during their evolution to the main sequence. Within the past $\sim 15$ years, several dozens of pulsating pre-MS stars were discovered using data obtained from ground and from space. Depending on their masses, pre-MS stars can show three different types of pulsations: (i) $\delta$ Scuti type p-mode pulsations, (ii) $\gamma$ Doradus like g-mode oscillations and (iii) g-mode Slowly Pulsating B star pulsations.

Our asteroseismic investigations yielded new insights into the connection between the pulsations and early stellar evolution: We revealed a relation between the stars' oscillatory behavior and their relative evolutionary stages that might lead us to a model-independent determination of the stars' fundamental parameters. With this we will be able to put constraints on theoretical models and help to answer some of the yet open questions in early stellar evolution.
\end{abstract}

Keywords. asteroseismology, stars: early type, stars: pre-main sequence, stars: variables: $\delta$ Scuti, stars: oscillations, stars: fundamental parameters

\section{Introduction}

Asteroseismology has advanced our understanding of stellar physics for a wide variety of stars across the Hertzsprung-Russel (HR) diagram. Data from space missions like MOST (Walker et al. 2003), CoRoT (Baglin 2006) and Kepler (Koch et al. 2010) allowed us to study the stars' interior structures in a way that has never been possible before. In some fields (e.g., for the red giant oscillators) the ultra precise data yielded big breakthroughs, while in other areas even more open questions were raised.

Since a few years now it is also possible to investigate the pulsational properties of the youngest stars: pre-main sequence (pre-MS) objects that gain their energy mainly from gravitational contraction and have not started hydrogen core burning yet.

A wealth of different types of pulsators are known across the HR diagram. They can be distinguished by the different excitation mechanisms (e.g., stochastic excitation or heat engine) driving their pulsations and by their restoring forces - pressure or buoyancy - that causes p- or g-modes respectively. But we can add another dimension to this distinction: the evolutionary stages, and hence stellar ages.

Going from the oldest to the youngest pulsating stars, we find the white dwarf and post-AGB pulsators to be the oldest members followed by clear post-main sequence (post-MS) stars like the red giant oscillators. Massive B type objects (e.g., $\beta$ Cephei stars) are definitely in their main sequence or early post-MS phase. Close to the zero-age main sequence (ZAMS) the distinction of pre-MS, ZAMS and early post-MS objects is ambiguous. As stars in these stages have similar atmospheres, observational parameters like effective temperature $\left(T_{\text {eff }}\right)$, gravity $(\log g)$, and even mass and luminosity are not 


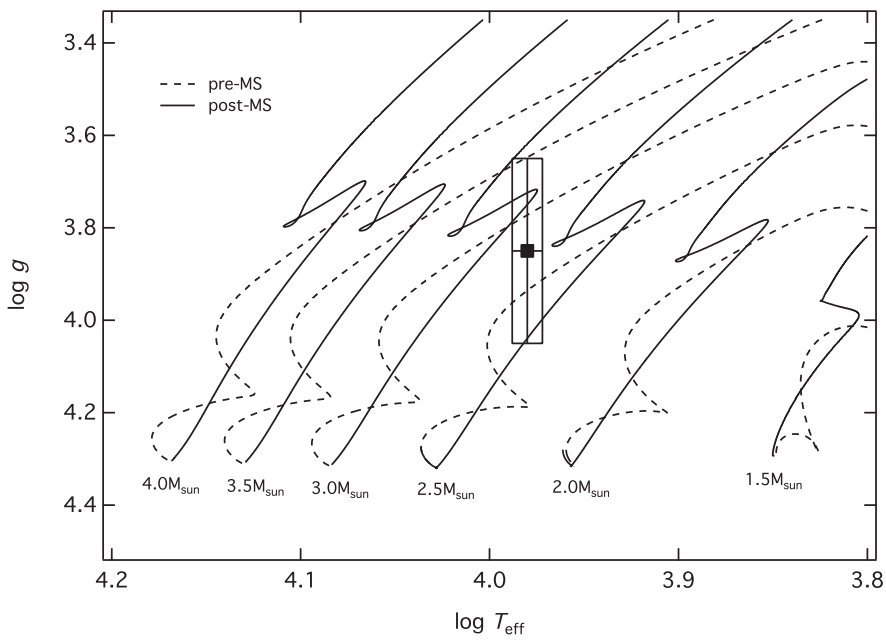

Figure 1. Intersecting pre- and post-MS evolutionary tracks (dashed and solid lines, respectively; Guenther et al. 2007 and Guenther \& Brown 2003) and the position of a random star illustrating its ambiguous evolutionary stage.

enough to distinguish the evolutionary phase, and hence, the stellar age. The intersecting pre- and post-MS evolutionary tracks close to the ZAMS shown in Figure 1 illustrate this problem. Therefore, a different method is needed to distinguish the stars' evolutionary stages.

\section{Pulsating pre-main sequence stars - a young research field}

Although the first two pulsating pre-MS stars - V588 Mon and V589 Mon - were already detected in 1972 (Breger 1972) in the young cluster NGC 2264, it took more than 20 years until the existence of this new group of stars was confirmed by the discovery of pulsation in the Herbig Ae field star HR 5999 and, subsequently, by the first seismic study of a pre-MS star (Kurtz \& Marang 1995). In the year 2000, only $8 \delta$ Scuti type pulsating pre-MS stars were known: four Herbig Ae stars and four cluster members. Back then it was assumed that pre-MS objects are purely radial pulsators showing only few pulsation modes (Marconi \& Palla 1998).

A comparison between the theoretical pulsation frequency spectra of pre- and postMS stars with same masses, effective temperatures and luminosities was conducted by Suran et al. (2001). The authors showed that the analysis of the oscillation frequency distributions allows to discriminate between the two evolutionary phases.

In subsequent years, it was observationally proven that pre-MS p-mode pulsators can be as multi-periodic as their more evolved (post-) MS counterparts and that they also show radial and non-radial oscillations (Zwintz et al. 2007). Using ground-based observations of the young cluster member NGC 6530 ZW278, its pulsation frequencies were successfully used to constrain the evolutionary stage of this pre-MS object only from asteroseismology (Guenther et al. 2007).

Due to dedicated observations, the number of $\delta$ Scuti type pre-MS stars increased significantly to 36 members until 2008 and enabled for the first time to investigate the instability region for pre-MS stars empirically (Zwintz 2008). The location of all known pre-MS pulsators in the HR-diagram suggested a pre-MS instability region that is slightly inclined toward the bluer (i.e., hotter) side relative to the classical instability strip under the plausible assumption that both instability regions coincide on the ZAMS. The biggest 


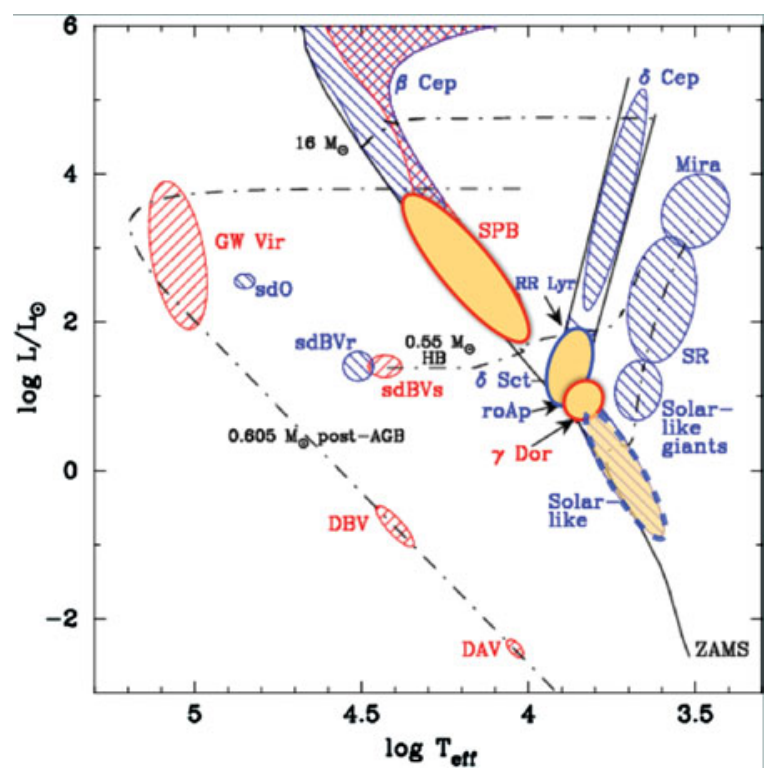

Figure 2. Different types of pre-MS pulsators in the HR diagram: the regions of pre-MS SPB, $\delta$ Scuti and $\gamma$ Doradus stars are marked as shaded areas with solid borders; the location of the theoretically predicted group of pre-MS solar like oscillators is indicated by a shaded area and a dashed border (Figure adapted from J Christensen-Dalsgaard).

challenge of this investigation was the lack of fundamental parameters determined from high-resolution spectroscopy. The only common measurements for all known stars of this group has been broad-band Johnson photometry. To improve on this, we successfully submitted several observing proposals in the following years to obtain high-resolution spectra of all known pre-MS pulsators and candidates.

\subsection{Types of pre-MS pulsators}

While about $60 \delta$ Scuti type pulsating pre-MS stars have been identified and well studied from ground and from space within the last decade, two new types of pre-MS pulsators were discovered only recently: the cooler pre-MS $\gamma$ Doradus type (Zwintz et al. 2013) and the hotter pre-MS Slowly Pulsating B (SPB) type stars (Gruber et al. 2012). While SPB pulsation is driven by the $\kappa$-mechanism, the excitation of $\gamma$ Doradus pulsation is caused by convective blocking (Guzik et al. 2000). Both groups are g-mode pulsators carrying information about deeper layers inside the stars compared to the p-mode $\delta$ Scuti oscillators. For both groups only two members each have been confirmed, while several more candidates are currently under investigation. The presence of $\delta$ Scuti - $\gamma$ Doradus hybrid pulsation in a pre-MS star was reported by Ripepi et al. (2011).

Figure 2 shows the positions of different types of pulsators in the HR diagram (adapted from J. Christensen-Dalsgaard) where the observed types of pre-MS oscillators - SPB, $\delta$ Scuti and $\gamma$ Doradus - are marked as shaded areas with solid borders. The group of pre-MS solar like oscillators (marked with a shaded area and a dashed border) has been theoretically predicted (Samadi et al. 2005), but no confirmed candidates have been discovered from observations yet.

\subsection{Observational data}

Photometric time series mostly have been obtained from the space telescopes MOST (Walker et al. 2003) and CoRoT (Baglin 2006). Earlier investigations are based on 


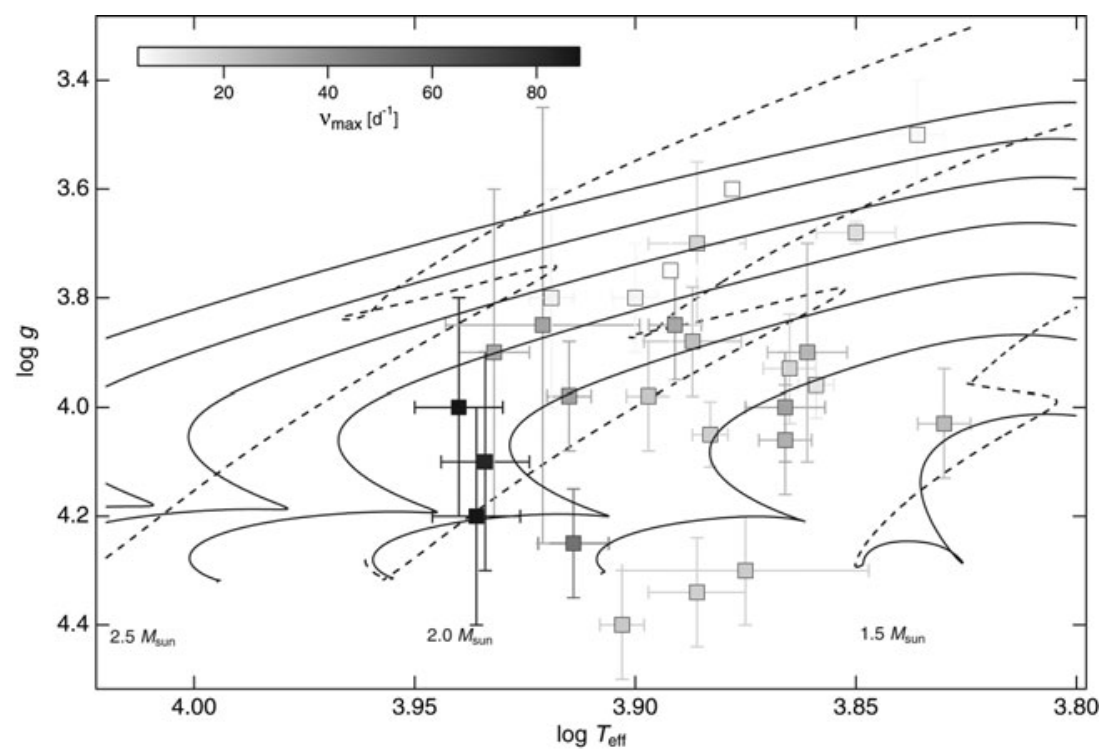

Figure 3. Kiel diagram showing 27 pre-MS $\delta$ Scuti stars for which time series photometry from space and fundamental parameters from high-resolution spectroscopy are available, color-coded with the frequency of maximum power, $\nu_{\max }$. Dashed lines show the pre-MS (Guenther et al. 2007) and solid lines post-MS evolutionary tracks (Guenther \& Brown 2003).

ground-based campaigns (e.g., Zwintz et al. 2007). Recently, the Kepler K2 mission observed some pre-MS objects as well.

For the determination of the stars' fundamental parameters we obtained high-resolution, high signal-to-noise spectroscopy at the McDonald $2.7 \mathrm{~m}$ telescope with the Tull spectrograph, the ESO VLT with UVES, the CFHT with ESPaDOnS, the ESO 3.6m telescope with HARPS, and the La Palma Mercator telescope with HERMES. For only few stars literature data were available (see Zwintz et al. 2014).

\section{Oscillation properties and stage in pre-MS evolution}

Recently, we revealed a relation between the oscillatory properties of heat-driven $\delta$ Scuti type pre-MS stars and the relative stage in their pre-MS evolution (Zwintz et al. 2014). We found that the the highest observed p-mode frequency, $f_{\text {max }}$, increases with the pre-MS stars' evolution from the birthline to the ZAMS. Or in other words: The further the pre-MS stars have progressed in their evolution towards the ZAMS, and hence the more compact they have become, the faster they oscillate (Zwintz et al. 2014).

To investigate this further, we conducted the same analysis using the frequency of maximum power, $\nu_{\max }$, and see a very similar relation (see Figure 3 ).

Relations like this have so far only been shown for the stochastically excited solarlike oscillations in red giants (e.g., Bedding et al. 2011) - a completely different type of pulsations in a different evolutionary stage.

We then investigated if the "classical" (post-) main sequence $\delta$ Scuti stars follow a similar relation than the one found for the pre-MS $\delta$ Scuti stars. For this we searched the literature for studies of (post-) main sequence stars of comparable quality to our data for the pre-MS pulsators, i.e., high precision time series photometry from space or from ground-based multi-site campaigns in combination with fundamental parameters from (high-resolution) spectroscopy. Our complete sample therefore comprises 23 (post-) 


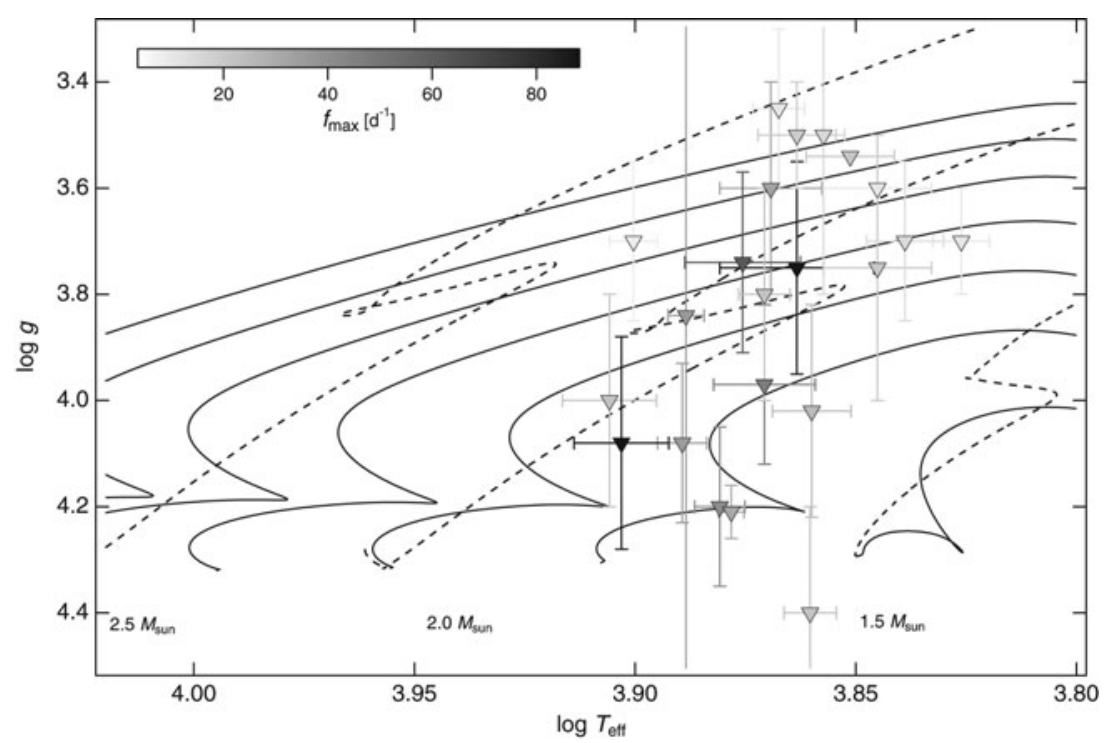

Figure 4. Kiel diagram showing 23 (post-) main sequence $\delta$ Scuti stars for which time series photometry from space or from multi-site campaigns and fundamental parameters from high-resolution spectroscopy are available, color-coded with the highest excited p-mode frequency $f_{\text {max }}$. Dashed lines show the pre-MS (Guenther et al. 2007) and solid lines post-MS evolutionary tracks (Guenther \& Brown 2003).

main sequence $\delta$ Scuti stars that were either subject of our own analyses or found in the literature. From Figure 4 it can clearly be seen that (post-) main sequence $\delta$ Scuti stars do not follow a relation like it was found for the pre-MS counterparts (Zwintz et al. 2014).

\section{Observations of pre-MS $\gamma$ Doradus and Slowly Pulsating B stars}

\subsection{Pre-MS $\gamma$ Doradus stars}

The existence of pre-MS $\gamma$ Doradus type stars with early F spectral types was suggested theoretically by Bouabid et al. (2011) without observational evidence. Using photometric time series obtained by the CoRoT space telescope (Baglin 2006) for stars in the young cluster NGC 2264 in combination with high-resolution spectroscopic measurements from ground, the first two pre-MS $\gamma$ Doradus stars have been discovered (Zwintz et al. 2013). Several other candidate members of NGC 2264 show similar properties and are currently under investigation. Figure 5 shows the location of the two bona-fide pre-MS $\gamma$ Doradus stars (Zwintz et al. 2013) and the three additional candidates in NGC 2264 in the Kiel diagram. Also shown is the location of the pre-MS $\delta$ Scuti - $\gamma$ Doradus hybrid pulsator CoID 102699796 (Ripepi et al. 2011). From this it can clearly be seen that only one star is located within the borders of the theoretically computed instability region for pre-MS $\gamma$ Doradus stars. These findings suggest that the theoretical calculations of the pre-MS $\gamma$ Doradus instability strip need to be revisited, now that members of this group of stars were identified observationally.

\subsection{Pre-MS Slowly Pulsating B (SPB) stars}

The presence of SPB type pulsations among pre-MS stars is currently discussed as two candidates have been reported in the young open cluster NGC 2244 (Gruber et al. 2012). 


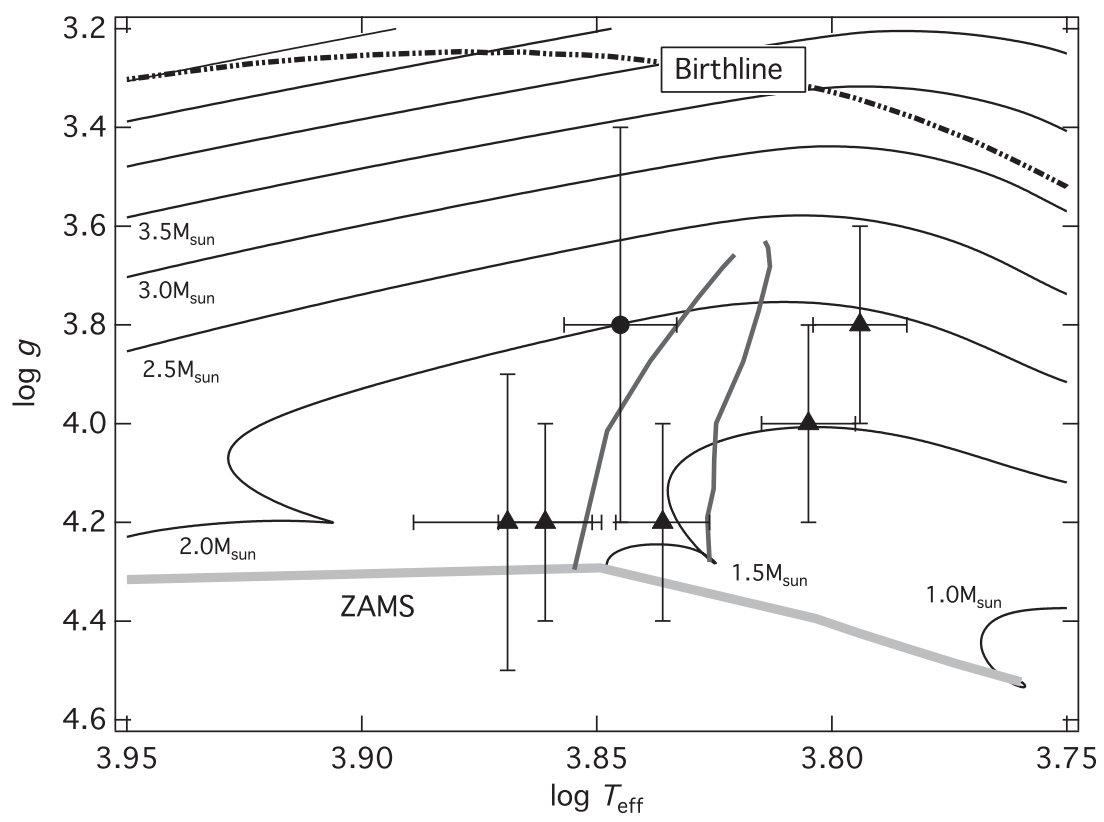

Figure 5. Location of the pre-MS $\gamma$ Doradus stars and candidates (solid triangles) and the confirmed pre-MS $\delta$ Scuti - $\gamma$ Doradus hybrid pulsator (solid circle) in the Kiel diagram. Pre-MS evolutionary tracks and the birthline (Guenther et al. 2007) are shown as solid and dashed-dotted lines, respectively, and the ZAMS is given in light grey.

(Pre-MS) SPB stars with $\sim 2$ to 7 solar masses are more massive than the (pre-MS) $\delta$ Scuti stars. Hence, they have a significantly shorter pre-MS phase lasting only less than 100000 years. Pre-MS SPB stars are already in the crucial transition phase from gravitational contraction to hydrogen-core burning where the star undergoes significant structural changes before arrival on the ZAMS.

Additional data for young B type objects are already available and under investigation from recent MOST (Walker et al. 2003) observations of the young cluster NGC 2264. The location of all potential pre-MS SPB stars in the Kiel diagram is shown in Figure 6.

\section{Conclusions and future prospects}

Asteroseismology of pre-MS stars has the potential to advance our understanding of early stellar evolution and to help us in the interpretation of the pulsational properties of main sequence and post-MS stars. We have been able to use the observed pulsation frequencies to determine the evolutionary stage of a $\delta$ Scuti star (Guenther et al. 2007). Furthermore, a relation was found between the highest excited p-mode frequency and the relative stage in the pre-MS evolution (Zwintz et al. 2014) which might build the basis for an independent measurement of pre-MS lifetimes and ages through asteroseismic methods.

A comparison to the more evolved (post-) main sequence $\delta$ Scuti stars showed that this relation must be connected to the early evolutionary stage, and is not a phenomenon common for $\delta$ Scuti pulsators during all phases of their evolution.

The discovery and investigation of new members of the groups of pre-MS $\gamma$ Doradus and pre-MS SPB type stars will help us to put observational constraints on the treatment of convection and to probe the interior structures of stars just before the energy 


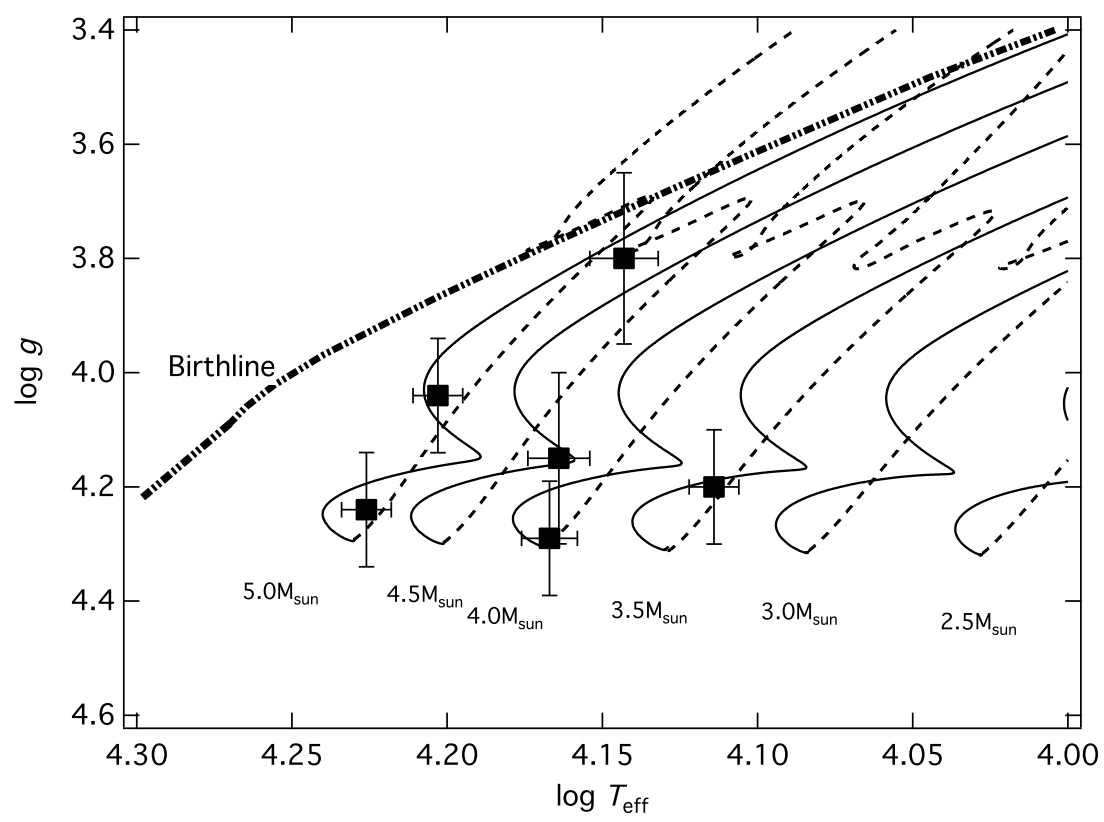

Figure 6. Location of the pre-MS SPB stars and candidates (solid squares) in the Kiel diagram. Pre-MS evolutionary tracks and the birthline (Guenther et al. 2007) are shown as solid and dashed-dotted lines, respectively.

production changes from gravitational contraction to full equilibrium hydrogen core burning, respectively.

On the observational side, new results are expected with longer photometric time series (i.e., longer than $\sim 40$ days from MOST or CoRoT) obtained from Kepler K2 or in the future using TESS (Ricker et al. 2014), BRITE-Constellation (Weiss et al. 2014) or PLATO (Rauer et al. 2014). But also the theoretical models for pre-MS objects need to be improved, e.g., in how rotation, convection and accretion are treated.

\section{References}

Baglin, A. 2006, in: M. Fridlund, A. Baglin, J. Lochard, \& L. Conroy (eds.), The CoRoT Mission, Pre-launch Status, Stellar Seismology and Planet Finding (ESASP 1306; Noordwijk, The Netherlands: ESA Publications Division)

Bouabid, M.-P., Montalban, J., Miglio, A., Dupret, M.-A., Grigahcène, A., \& Noels, A. 2011, $A \mathscr{E} A, 531,145$

Breger, M. 1972, ApJ, 171, 539

Gruber, D., Saio, H., Kuschnig, R., et al. 2012, MNRAS, 420, 291

Guenther, D. B. \& Brown, K. I. T. 2003, ApJ, 600, 419

Guenther, D. B., Kallinger, T., Zwintz, K., Weiss, W. W., \& Tanner, J. 2007, ApJ, 671, 581

Guzik, J. A., Kaye, A. B., Bradley, P., Cox, A. N., \& Neuforge, C. 2000, ApJ, 542, 57

Koch, D. G., Borucki, W. J., Basri, G., et al. 2010 2010, ApJ, 713, L79

Kurtz, D. \& Marang, F. 1995, MNRAS, 276, 191

Marconi, M. \& Palla, F. 1998, AJ, 507, 141

Rauer, H., Catala, C., Aerts, C., et al. 2014, Experimental Astronomy, 38, 249

Ricker, G. R., Winn, J. N., Vanderspek, R., et al. 2014, in: Proceedings of the SPIE, Volume 9143, id. 914320

Ripepi, V., Cusano, F., de Criszenzo, M., et al. 2011, MNRAS, 416, 1535 
Samadi, R., Goupil, M.-J., Alecian, E., Baudin, F., Georgobiani, D. Trampedach, R., Stein, R., \& Nordlund, A. 2005, Journal of Astrophysics and Astronomy, 26, 171

Suran, M., Goupil, M., Baglin, A., Lebreton, Y., \& Catala, C. 2001, A\&A, 372, 233

Walker, G. A. G., Matthews, J. M., Kuschnig, R., et al. 2003, PASP, 115, 1023

Weiss, W. W., Rucinski, S. M., Moffat, A. F. J., et al. 2014, PASP, 126, 573

Zwintz, K., Guenther, D. B., \& Weiss, W. W. 2007, ApJ, 655, 342

Zwintz, K. 2008, ApJ, 673, 1088

Zwintz, K., Fossati, L., Ryabchikova, A. et al. 2013, A\&\&A, 550, 121

Zwintz, K., Fossati, L., Ryabchikova, A. et al. 2014, Science, 345, 550 